Mathematical Modelling and Analysis

Volume 19 Number 4, September 2014, 537-555

http://dx.doi.org/10.3846/13926292.2014.960016

(c) Vilnius Gediminas Technical University, 2014
Publisher: Taylor\&Francis and VGTU

http://www.tandfonline.com/TMMA

Print ISSN: 1392-6292

Online ISSN: 1648-3510

\title{
Existence of Solitary Waves and Periodic Waves to a Perturbed Generalized KdV Equation*
}

\author{
Weifang Yan $^{a}$, Zhengrong $\mathrm{Liu}^{b}$ and Yong Liang ${ }^{b}$ \\ ${ }^{a}$ School of Mathematics and Statistics Science, Ludong University \\ 264025 Yantai, China \\ ${ }^{b}$ Department of Mathematics, South China University of Technology \\ 510640 Guangzhou, China \\ E-mail(corresp.): yan.wf@mail.scut.edu.cn \\ E-mail: liuzhr@scut.edu.cn \\ E-mail: dyliang@scut.edu.cn
}

Received October 25, 2013; revised August 25, 2014; published online September 1, 2014

\begin{abstract}
In this paper, the existence of solitary waves and periodic waves to a perturbed generalized KdV equation is established by applying the geometric singular perturbation theory and the regular perturbation analysis for a Hamiltonian system. Moreover, upper and lower bounds of the limit wave speed are obtained. Some previous results are extended.

Keywords: perturbed generalized KdV equation, traveling waves, geometric singular perturbation theory, regular perturbation analysis for a Hamiltonian system.
\end{abstract}

AMS Subject Classification: 34F10; 35B10; 35B44; 35C07.

\section{Introduction}

Many nonlinear wave equations have been presented in fields such as physics, chemistry, biology, mechanics, optics, etc. For a long time, the KdV equation has attracted much attention due to its significant nature in physical contexts, stratified internal waves, ion-acoustic waves, plasma physics $[1,11]$. There exists a lot of investigation on explicit and exact solitary wave solutions to the $\mathrm{KdV}$ equation, for instance, $[2,8,9]$. Recently, many researchers have paid attention to the perturbed and generalized $\mathrm{KdV}$ equation, for example, $[3,4,5,7,10,13$, $14,15,16,17,18,19]$.

In this paper, we consider the perturbed generalized KdV equation

$$
u_{t}+u^{n} u_{x}+u_{x x x}+\varepsilon\left(u_{x x}+u_{x x x x}\right)=0,
$$

* Research is supported by the National Natural Science Foundation of China (No. 11171115) and the Fundamental Research Funds for the Central Universities (No. 2013ZM0115). 
where $n>0$ is an integer and $\varepsilon>0$ is a perturbation parameter. Clearly, when $\varepsilon=0$, Eq. (1.1) becomes the generalized KdV equation

$$
u_{t}+u^{n} u_{x}+u_{x x x}=0 .
$$

When $n=1$, Eq. (1.1) and (1.2) are reduced to the perturbed KdV equation

$$
u_{t}+u u_{x}+u_{x x x}+\varepsilon\left(u_{x x}+u_{x x x x}\right)=0,
$$

and the $\mathrm{KdV}$ equation

$$
u_{t}+u u_{x}+u_{x x x}=0,
$$

respectively. Ogawa [17] solved two problems for Eq. (1.3). One is the persistence of solitary waves and periodic waves, and the other is the wave speed selection principle. It is well known that for arbitrary positive wave speed $c$, Eq. (1.4) has a solitary wave solution

$$
u(x, t)=3 c \operatorname{sech}^{2} \frac{\sqrt{c}}{2}(x-c t) .
$$

However, Ogawa [17] showed that for each sufficiently small $\varepsilon>0$, Eq. (1.3) has only one solitary wave solution with a uniquely determined speed. These solitary wave solutions converge to the solitary wave solution of the KdV equation with a uniquely determined limit speed as $\varepsilon$ tends to zero.

In this paper, our purpose is to extend Ogawa's results [17] to Eq. (1.1). The remaining part is organized as follows. Section 2 is devoted to some preliminary discussion and the main theorem. In Section 3, the geometric singular perturbation theory and the regular perturbation analysis for a Hamiltonian system are used to investigate the existence of solitary wave solutions and periodic wave solutions of Eq. (1.1). In Section 4, the Abelian integral theory is used to analysis the limit speed, and the implicit function theorem is applied to prove our main theorem. This paper ends with a brief conclusion.

\section{Our Main Result}

In this section, we state the main result of this paper.

For given constant $c>0$, substituting $u=\varphi(\xi)$ with $\xi=x-c t$ into (1.1), it follows that

$$
-c \varphi^{\prime}(\xi)+\varphi^{n} \varphi^{\prime}(\xi)+\varphi^{\prime \prime \prime}(\xi)+\varepsilon\left(\varphi^{\prime \prime}(\xi)+\varphi^{\prime \prime \prime \prime}(\xi)\right)=0 .
$$

Integrating this equation and setting the integral constant to be zero, we have

$$
-c \varphi(\xi)+\frac{1}{n+1} \varphi^{n+1}(\xi)+\varphi^{\prime \prime}(\xi)+\varepsilon\left(\varphi^{\prime}(\xi)+\varphi^{\prime \prime \prime}(\xi)\right)=0 .
$$

Moreover if we perform the scale transformations $\varphi=\sqrt[n]{c} \psi$ and $\xi=\tau / \sqrt{c}$ to (2.2), the final equation is given as

$$
-\psi(\tau)+\frac{1}{n+1} \psi^{n+1}(\tau)+\psi^{\prime \prime}(\tau)+\varepsilon\left(\frac{1}{\sqrt{c}} \psi^{\prime}(\tau)+\sqrt{c} \psi^{\prime \prime \prime}(\tau)\right)=0 .
$$


If we obtain a solution $\psi(\tau)$ of (2.3) for some $\varepsilon>0$ and $c>0$, then the corresponding $\varphi(\xi)$ is a solution of (2.1), that is, a traveling wave solution to the original equation (1.1).

Now the unperturbed equation of (2.3) is

$$
-\psi(\tau)+\frac{1}{n+1} \psi^{n+1}(\tau)+\psi^{\prime \prime}(\tau)=0
$$

whose solutions are traveling wave solutions to the generalized $\mathrm{KdV}$ equation (1.2) under the above scale transformations. (2.4) has an equivalent form

$$
\left\{\begin{array}{l}
\frac{\mathrm{d} \psi}{\mathrm{d} \tau}=y \\
\frac{\mathrm{d} y}{\mathrm{~d} \tau}=\psi-\frac{1}{n+1} \psi^{n+1}
\end{array}\right.
$$

which is a Hamiltonian system with the Hamiltonian function

$$
H(\psi, y)=-\frac{1}{2} y^{2}+\frac{1}{2} \psi^{2}-\frac{1}{(n+1)(n+2)} \psi^{n+2} .
$$

We consider the phase portrait of system (2.5). Firstly, consider the case of $n$ is even, it is easy to see that system (2.5) has three singular points $(0,0)$ and $( \pm \sqrt[n]{n+1}, 0) .(0,0)$ is a saddle point and $( \pm \sqrt[n]{n+1}, 0)$ are two centers. Consider a level curve of the form $H=h$. It corresponds to two periodic orbits if $h$ satisfies $0<h<\left(n \sqrt[n]{(n+1)^{2}}\right) /(2(n+2))$. If $h=0$, then it includes two homoclinic orbits connecting with $(0,0)$. If $h<0$, then it represents a big periodic orbit surrounding the three singular points. The phase portrait of system (2.5) is given as Fig. 1(a).

Secondly, consider the case of $n$ is odd. System (2.5) has two singular points $(0,0)$ and $(\sqrt[n]{n+1}, 0) .(0,0)$ is a saddle point and $(\sqrt[n]{n+1}, 0)$ is a center. Consider a level curve of the form $H=h$. It corresponds to a periodic orbit if $h$ satisfies $0<h<n(n+1)^{\frac{2}{n}} / 2(n+2)$. If $h=0$, then it includes a homoclinic orbit and an open orbit connecting with $(0,0)$. If $h<0$, then it represents an open orbit. The phase portrait of system (2.5) is given as Fig. 1(b). Therefore we can parameterize all solutions of $(2.4)$ by $h$. Further, using this parametrization, we can describe the existence result of solitary wave solutions and periodic wave solutions of (1.1) as Theorem 1. The proof will be given in Section 3 and Section 4.

Theorem 1. Consider Eq. (1.1). For given positive integer n, let

$$
h_{n}=\frac{n \sqrt[n]{(n+1)^{2}}}{2(n+2)}
$$

(1) If $n$ is even, then there exists $\varepsilon_{n}^{*}>0$ such that for each $\varepsilon \in\left(0, \varepsilon_{n}^{*}\right)$ and $h \in\left[0, h_{n}\right), E q$. (1.1) has two traveling wave solutions

$$
u_{ \pm}= \pm \sqrt[n]{c} \psi(\varepsilon, h, c, \tau)
$$




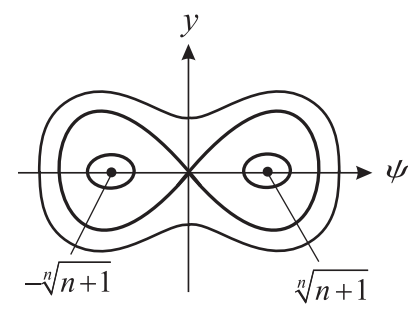

(a) $n$ is even

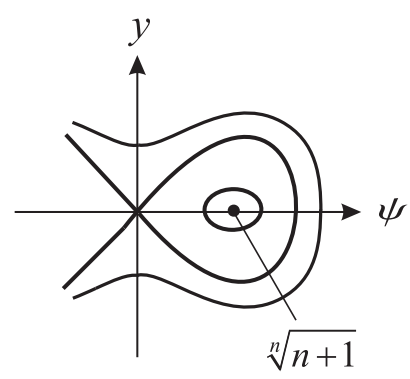

(b) $n$ is odd

Figure 1. The phase portrait of system (2.5).

where $c=c(\varepsilon, h)$, and $\psi(\varepsilon, h, c, \tau)$ is a solution of Eq. (2.3) satisfying

$$
\frac{\partial}{\partial \tau} \psi(\varepsilon, h, c, 0)=0, \quad \frac{\partial^{2}}{\partial \tau^{2}} \psi(\varepsilon, h, c, 0)<0 .
$$

(2) If $n$ is odd, then for each $\varepsilon \in\left(0, \varepsilon_{n}^{*}\right)$ and $h \in\left[0, h_{n}\right)$, Eq. (1.1) has a traveling wave solution $u_{+}$given in (2.8).

(3) When $h=0, u_{+}$and $u_{-}$are two solitary wave solutions. When $h \in$ $\left(0, h_{n}\right), u_{+}$and $u_{-}$are two periodic wave solutions.

(4) $c(\varepsilon, h)$ is a smooth function of $\varepsilon$ and $h$. When $\varepsilon \rightarrow 0, c(\varepsilon, h)$ converges to $c_{0}(h)$, where $c_{0}(h)$ is a smooth function for $h \in\left[0, h_{n}\right)$ and satisfies

$$
\frac{1}{n} \leq c_{0}(h) \leq \frac{3 n+4}{2 n^{2}+3 n}, \quad c_{0}(0)=\frac{3 n+4}{2 n^{2}+3 n}, \quad \lim _{h \rightarrow h_{n}-0} c_{0}(h)=\frac{1}{n} .
$$

(5) When $\varepsilon \rightarrow 0, \psi(\varepsilon, h, c, \tau)$ converges to $\psi\left(h, c_{0}(h), \tau\right)$ uniformly in $\tau$, where $\psi\left(h, c_{0}(h), \tau\right)$ is a solution of system (2.5) on the level curve $H=h$ and satisfies

$$
\frac{\partial}{\partial \tau} \psi\left(h, c_{0}(h), 0\right)=0, \quad \frac{\partial^{2}}{\partial \tau^{2}} \psi\left(h, c_{0}(h), 0\right)<0 .
$$

\section{Perturbation Analysis}

In this section, we use the geometric singular perturbation theory and the regular perturbation analysis for a Hamiltonian system to investigate the existence of solitary wave solutions and periodic wave solutions of Eq. (1.1). We only consider the case of $n$ is even, since the case of $n$ is odd is similar.

We first introduce the following result on invariant manifolds which is due to Fenichel [6]. For convenience, we use a version of this theorem due to Jones [12].

Lemma 1 [Geometric Singular Perturbation Theorem]. Consider the system

$$
\left\{\begin{array}{l}
x^{\prime}=f(x, y, \varepsilon), \\
y^{\prime}=\varepsilon g(x, y, \varepsilon),
\end{array}\right.
$$


where $x \in \mathbb{R}^{n}, y \in \mathbb{R}^{l}$ and $\varepsilon$ is a real parameter, $f, g$ are $C^{\infty}$ on the set $V \times I$, where $V \subset \mathbb{R}^{n+l}$ and $I$ is an open interval containing 0 . Assume that for $\varepsilon=0$, the system has a compact normally hyperbolic manifold $M_{0}$ which is contained in the set $f(x, y, 0)=0$. The manifold $M_{0}$ is said to be normally hyperbolic if the linearization of (3.1) at each point in $M_{0}$ has exactly l eigenvalues on the imaginary axis $\mathcal{R}(\lambda)=0$. Then for any $0<r<+\infty$, if $\varepsilon>0$ sufficiently small, there exists a manifold $M_{\varepsilon}$ such that the following conclusions hold.

(1) $M_{\varepsilon}$ is locally invariant under the flow of (3.1).

(2) $M_{\varepsilon}$ is $C^{r}$ in $x, y$ and $\varepsilon$.

(3) $M_{\varepsilon}=\left\{(x, y) \mid x=h^{\varepsilon}(y)\right\}$ for some $C^{r}$ function $h^{\varepsilon}$ and $y$ in some compact set $K$.

(4) There exist locally invariant stable and unstable manifolds $W^{s}\left(M_{\varepsilon}\right), W^{u}\left(M_{\varepsilon}\right)$, that lie within $\mathcal{O}(\varepsilon)$ of, and are diffeomorphic to $W^{s}\left(M_{0}\right)$ and $W^{u}\left(M_{0}\right)$.

Now let us go back to (2.3). Its equivalent form is

$$
\left\{\begin{array}{l}
\frac{\mathrm{d} \psi}{\mathrm{d} \tau}=y, \quad \frac{\mathrm{d} y}{\mathrm{~d} \tau}=z \\
\varepsilon \sqrt{c} \frac{\mathrm{d} z}{\mathrm{~d} \tau}=\psi-\frac{1}{n+1} \psi^{n+1}-z-\frac{\varepsilon}{\sqrt{c}} y .
\end{array}\right.
$$

For $\varepsilon>0,(3.2)$ defines a system of ODEs whose solutions evolve in the threedimensional $(\psi, y, z)$ phase space. In this phase space, there are critical points

$$
(0,0,0) \text { and }( \pm \sqrt[n]{n+1}, 0,0)
$$

Note that when $\varepsilon=0,(3.2)$ does not define a dynamical system in $\mathbb{R}^{3}$. This problem may be overcome by the transformation $\tau=\varepsilon \sigma$, under which the system becomes

$$
\left\{\begin{array}{l}
\frac{\mathrm{d} \psi}{\mathrm{d} \sigma}=\varepsilon y, \quad \frac{\mathrm{d} y}{\mathrm{~d} \sigma}=\varepsilon z, \\
\sqrt{c} \frac{\mathrm{d} z}{\mathrm{~d} \sigma}=\psi-\frac{1}{n+1} \psi^{n+1}-z-\frac{\varepsilon}{\sqrt{c}} y .
\end{array}\right.
$$

Generally, system (3.2) is referred to as the slow system since the time scale $\tau$ is slow. And system (3.3) is referred to as the fast system since the time scale $\sigma$ is fast. The two systems are equivalent when $\varepsilon>0$.

Consider the slow system (3.2). For $\varepsilon=0$, the flow is confined to the set

$$
M_{0}=\left\{(\psi, y, z) \in \mathbb{R}^{3}: z=\psi-\frac{1}{n+1} \psi^{n+1}\right\},
$$

which is a two-dimensional invariant manifold for system (3.2). If $M_{0}$ is normally hyperbolic, then for $\varepsilon>0$ sufficiently small, Lemma 1 provides us with a locally invariant manifold $M_{\varepsilon}$ for system (3.2). On this manifold consider system (3.2). The dimensionality is reduced back to two. 
In fact, to check that the invariant manifold $M_{0}$ is normally hyperbolic, we need to check that the linearization of the fast system (3.3), restricted to $M_{0}$, has precisely $\operatorname{dim} M_{0}$ eigenvalues on the imaginary axis, with the remainder of the spectrum being hyperbolic. The linearization of (3.3) restricted to $M_{0}$ is given by the following matrix

$$
A=\left(\begin{array}{ccc}
0 & 0 & 0 \\
0 & 0 & 0 \\
\frac{1}{\sqrt{c}}\left(1-\psi^{n}\right) & 0 & -\frac{1}{\sqrt{c}}
\end{array}\right),
$$

which has eigenvalues 0,0 and $-1 / \sqrt{c}$. Thus, $M_{0}$ is normally hyperbolic. By Lemma 1, there exists a locally invariant two-dimensional manifold $M_{\varepsilon}$ with $\varepsilon>0$ sufficiently small, under the flow of system (3.2), which can be written in the form

$$
M_{\varepsilon}=\left\{(\psi, y, z) \in \mathbb{R}^{3}: z=\psi-\frac{1}{n+1} \psi^{n+1}+\zeta(\psi, y, \varepsilon)\right\},
$$

where $\zeta(\psi, y, \varepsilon)$ depends smoothly on $\varepsilon$ and satisfies $\zeta(\psi, y, 0)=0$. Since $M_{0}$ is smooth, the vector field in (3.3) is smooth and $M_{\varepsilon}$ can be characterized as the graph of a function, we can expand $\zeta(\psi, y, \varepsilon)$ in $\varepsilon$ for $\varepsilon>0$ sufficiently small, that is,

$$
z=\psi-\frac{1}{n+1} \psi^{n+1}+\varepsilon \zeta_{1}(\psi, y)+\mathcal{O}\left(\varepsilon^{2}\right) .
$$

Substituting it into the last equation of the slow system (3.2), we get

$$
\varepsilon \sqrt{c}\left(1-\psi^{n}\right) y+\mathcal{O}\left(\varepsilon^{2}\right)=-\varepsilon \zeta_{1}(\psi, y)-\frac{\varepsilon}{\sqrt{c}} y+\mathcal{O}\left(\varepsilon^{2}\right) .
$$

Comparing coefficients of $\varepsilon$ yields

$$
\zeta_{1}(\psi, y)=\sqrt{c}\left(\psi^{n} y-\left(1+\frac{1}{c}\right) y\right)
$$

Thus, the dynamics on the slow manifold $M_{\varepsilon}$ is given as

$$
\left\{\begin{array}{l}
\frac{\mathrm{d} \psi}{\mathrm{d} \tau}=y \\
\frac{\mathrm{d} y}{\mathrm{~d} \tau}=\psi-\frac{1}{n+1} \psi^{n+1}+\varepsilon \sqrt{c}\left(\psi^{n} y-\left(1+\frac{1}{c}\right) y\right)+\mathcal{O}\left(\varepsilon^{2}\right) .
\end{array}\right.
$$

Now we can check if a periodic orbit persist or not as follows. By symmetry, we only need to check the orbit with $\psi>0$. Firstly, remember the dynamics of the unperturbed system (2.5), which can be understood by the level curve of $H$. Fix an initial data $(\alpha, 0)$ with $0<\alpha<\sqrt[n]{n+1}$. Now let $(\psi(\tau), y(\tau))$ be the solution of $(3.6)$ with $(\psi, y)(0)=(\alpha, 0)$. Then there exist $\tau_{1}>0$ and $\tau_{2}<0$ such that

$$
y(\tau)>0 \quad \text { for } 0<\tau<\tau_{1}, \quad y\left(\tau_{1}\right)=0
$$

and

$$
y(\tau)<0 \quad \text { for } \tau_{2}<\tau<0, \quad y\left(\tau_{2}\right)=0 .
$$


Let us define a function $\Phi$ as follows.

$$
\Phi(\alpha, c, \varepsilon)=\int_{\tau_{2}}^{\tau_{1}} \dot{H}(\psi, y) \mathrm{d} \tau
$$

where $\cdot$ denotes the derivative by $\tau, H(\psi, y)$ is defined as $(2.6)$, and the integral is performed along the orbit of (3.6). Hence

$$
\dot{H}(\psi, y)=\varepsilon \sqrt{c}\left(\psi^{n} y^{2}-\left(1+\frac{1}{c}\right) y^{2}\right)+\mathcal{O}\left(\varepsilon^{2}\right) .
$$

Note that $\Phi(\alpha, c, \varepsilon)$ denotes difference of the level between the two points on the $\psi$ axis:

$$
\Phi(\alpha, c, \varepsilon)=H\left(\psi\left(\tau_{1}\right), y\left(\tau_{1}\right)\right)-H\left(\psi\left(\tau_{2}\right), y\left(\tau_{2}\right)\right) .
$$

Therefore, $\Phi(\alpha, c, \varepsilon)=0$ if and only if $\psi(\tau)$ is a periodic solution of (3.6). And our aim is to solve $\Phi=0$. Since $\Phi(\alpha, c, 0)=0$, we have

$$
\Phi(\alpha, c, \varepsilon)=\varepsilon \tilde{\Phi}(\alpha, c, \varepsilon) .
$$

Now our aim is to solve $\tilde{\Phi}=0$. Note that when $\varepsilon \rightarrow 0, \tilde{\Phi}(\alpha, c, \varepsilon)$ has a limit

$$
\tilde{\Phi}_{0}(\alpha, c)=\lim _{\varepsilon \rightarrow 0} \tilde{\Phi}(\alpha, c, \varepsilon)=\sqrt{c} \int\left(\left(\psi_{0}^{n}-1\right) y_{0}^{2}-\frac{1}{c} y_{0}^{2}\right) \mathrm{d} \tau .
$$

Here, $\left(\psi_{0}, y_{0}\right)$ is a solution of (2.5) and this integral is performed on a level curve $H=H(\alpha, 0) \in\left(0, h_{n}\right)$. In the rest of this paper, for simplicity, we will omit some upper and lower limits of integral. Since

$$
\int \psi_{0}^{n} y_{0}^{2} \mathrm{~d} \tau=\int \psi_{0}^{n} \psi_{0}^{\prime 2} \mathrm{~d} \tau=\int \psi_{0}^{n} \psi_{0}^{\prime} \mathrm{d} \psi_{0}=-n \int \psi_{0}^{n} y_{0}^{2} \mathrm{~d} \tau-\int \psi_{0}^{n+1} \psi_{0}^{\prime \prime} \mathrm{d} \tau
$$

i.e.,

$$
\int \psi_{0}^{n} y_{0}^{2} \mathrm{~d} \tau=-\frac{1}{n+1} \int \psi_{0}^{n+1} \psi_{0}^{\prime \prime} \mathrm{d} \tau
$$

Similarly,

$$
\int y_{0}^{2} \mathrm{~d} \tau=-\int \psi_{0} \psi_{0}^{\prime \prime} \mathrm{d} \tau
$$

The above two equations yields

$$
\int\left(\psi_{0}^{n}-1\right) y_{0}^{2} \mathrm{~d} \tau=\int\left(\psi_{0}-\frac{1}{n+1} \psi_{0}^{n+1}\right) \psi_{0}^{\prime \prime} \mathrm{d} \tau=\int \psi_{0}^{\prime \prime 2} \mathrm{~d} \tau .
$$

Therefore

$$
\tilde{\Phi}_{0}(\alpha, c)=\sqrt{c} \int\left(\psi_{0}^{\prime \prime 2}-\frac{1}{c} \psi_{0}^{\prime 2}\right) \mathrm{d} \tau=\frac{1}{\sqrt{c}}\left(c \int \psi_{0}^{\prime \prime 2} \mathrm{~d} \tau-\int \psi_{0}^{\prime 2} \mathrm{~d} \tau\right) .
$$

And the limit speed $c_{0}$ is determined by

$$
c_{0} \int \psi_{0}^{\prime \prime 2} \mathrm{~d} \tau-\int \psi_{0}^{\prime 2} \mathrm{~d} \tau=0
$$


We can define the similar function for a homoclinic orbit as

$$
\Psi(c, \varepsilon)=\int_{-\infty}^{0} \dot{H}(\psi, y) \mathrm{d} \tau+\int_{0}^{\infty} \dot{H}(\psi, y) \mathrm{d} \tau .
$$

Here the former part is integrated along a solution $(\psi(\tau), y(\tau))$ on the one dimensional unstable manifold of the origin with $y(\tau)>0$ for $-\infty<\tau<0$ and $y(0)=0$. The latter is similar. $\tilde{\Psi}(c, \varepsilon)$ and $\tilde{\Psi}_{0}(c)$ are also defined similarly,

$$
\tilde{\Psi}(c, \varepsilon)=\frac{1}{\varepsilon} \Psi(c, \varepsilon), \quad \tilde{\Psi}_{0}(c)=\lim _{\varepsilon \rightarrow 0} \tilde{\Psi}(c, \varepsilon) .
$$

Consequently, we get

$$
\tilde{\Psi}_{0}(c)=\frac{1}{\sqrt{c}}\left(c \int \psi_{0}^{\prime \prime 2} \mathrm{~d} \tau-\int \psi_{0}^{\prime 2} \mathrm{~d} \tau\right),
$$

where $\psi_{0}$ is a solution of (2.5) and the integration is performed on the curve $H=0$, more precisely, on the homoclinic orbit of (2.5). Therefore the condition for the limit speed is the same as (3.8).

Remark 1. When $n$ is odd, by the same method, we can obtain the condition for the limit speed is the same as (3.8).

\section{Analysis by the Abelian Integral Theory}

In this section we concentrate ourselves on studying the property of the limit speed $c_{0}$ with $h$ and prove our main theorem. We assume $\psi$ is a solution of (2.4).

Firstly, let $Q$ and $R$ be

$$
Q=\frac{1}{2} \int \psi^{\prime \prime 2} \mathrm{~d} \tau, \quad R=\frac{1}{2} \int \psi^{\prime 2} \mathrm{~d} \tau
$$

And when $0 \leq k<k_{n}=2 h_{n}$, let the two non-negative real roots of

$$
\psi^{2}-\frac{2}{(n+1)(n+2)} \psi^{n+2}=k
$$

be $\alpha(k)$ and $\beta(k)$ such that $0 \leq \alpha(k)<\beta(k)$. As mentioned above, the orbit $(\psi(\tau), y(\tau))$ is on the level curve $H=h=k / 2$, where $y=\mathrm{d} \psi / \mathrm{d} \tau$. Therefore we have

$$
Q=\int_{\alpha}^{\beta} \frac{\left(\psi-\frac{1}{n+1} \psi^{n+1}\right)^{2}}{E(\psi)} \mathrm{d} \psi, \quad R=\int_{\alpha}^{\beta} E(\psi) \mathrm{d} \psi,
$$

by system (2.5). Here,

$$
E(\psi)=\sqrt{\psi^{2}-\frac{2}{(n+1)(n+2)} \psi^{n+2}-k} .
$$

Now $Q$ and $R$ are the functions of only $k$. We will prove the following proposition in Lemma 2-11. And this will assert the property of the limit speed $c_{0}$ with $k$. 
Proposition 1. Let $X(k)=\frac{Q}{R}$. Then we have

$$
\frac{2 n^{2}+3 n}{3 n+4}<X(k)<n \quad \text { for } \quad 0<k<k_{n} .
$$

Moreover

$$
X(0)=\frac{2 n^{2}+3 n}{3 n+4} \quad \text { and } \quad \lim _{k \rightarrow k_{n}-0} X(k)=n .
$$

It is convenient to represent $Q$ and $R$ by the following integrals:

$$
J_{m}(k)=\int_{\alpha}^{\beta} \psi^{m} E(\psi) \mathrm{d} \psi, \quad m=0,1,2, \ldots
$$

Note that

$$
\int_{\alpha}^{\beta} \frac{\psi^{m}}{E(\psi)} \mathrm{d} \psi=-2 J_{m}^{\prime}(k) .
$$

Therefore $Q$ and $R$ are represented as follows:

$$
Q=-2 J_{2}^{\prime}+\frac{4}{n+1} J_{n+2}^{\prime}-\frac{2}{(n+1)^{2}} J_{2 n+2}^{\prime}, \quad R=J_{0} .
$$

We have the following two lemmas.

Lemma 2. Let $B(p, q)=\int_{0}^{1} x^{p-1}(1-x)^{q-1} \mathrm{~d} x, p>0, q>0$ be the Beta function. Then we have

$$
\begin{gathered}
J_{0}(0)=\frac{1}{n}\left(\frac{(n+1)(n+2)}{2}\right)^{\frac{2}{n}} B\left(\frac{3}{2}, \frac{2}{n}\right), \\
J_{n}(0)=\frac{1}{n}\left(\frac{(n+1)(n+2)}{2}\right)^{\frac{n+2}{n}} B\left(\frac{3}{2}, \frac{n+2}{n}\right),
\end{gathered}
$$

and

$$
\frac{J_{n}(0)}{J_{0}(0)}=\frac{2(n+1)(n+2)}{3 n+4} .
$$

Proof. Since $\alpha(0)=0, \beta(0)=\sqrt[n]{\frac{(n+1)(n+2)}{2}}$, we get

$$
J_{0}(0)=\int_{0}^{\sqrt[n]{\frac{(n+1)(n+2)}{2}}} \psi \sqrt{1-\frac{2}{(n+1)(n+2)} \psi^{n}} \mathrm{~d} \psi,
$$

and

$$
J_{n}(0)=\int_{0}^{\sqrt[n]{\frac{(n+1)(n+2)}{2}}} \psi^{n+1} \sqrt{1-\frac{2}{(n+1)(n+2)} \psi^{n}} \mathrm{~d} \psi .
$$

Let $1-\frac{2}{(n+1)(n+2)} \psi^{n}=t$. Then we obtain

$$
\begin{aligned}
J_{0}(0) & =\frac{1}{n}\left(\frac{(n+1)(n+2)}{2}\right)^{\frac{2}{n}} \int_{0}^{1} t^{\frac{1}{2}}(1-t)^{\frac{2}{n}-1} \mathrm{~d} t \\
& =\frac{1}{n}\left(\frac{(n+1)(n+2)}{2}\right)^{\frac{2}{n}} B\left(\frac{3}{2}, \frac{2}{n}\right),
\end{aligned}
$$


and

$$
\begin{aligned}
J_{n}(0) & =\frac{1}{n}\left(\frac{(n+1)(n+2)}{2}\right)^{\frac{n+2}{n}} \int_{0}^{1} t^{\frac{1}{2}}(1-t)^{\frac{2}{n}} \mathrm{~d} t \\
& =\frac{1}{n}\left(\frac{(n+1)(n+2)}{2}\right)^{\frac{n+2}{n}} B\left(\frac{3}{2}, \frac{n+2}{n}\right) .
\end{aligned}
$$

Since

$$
B(p, q)=\frac{\Gamma(p) \Gamma(q)}{\Gamma(p+q)}, \quad \Gamma(s+1)=s \Gamma(s),
$$

where $\Gamma(s)=\int_{0}^{+\infty} x^{s-1} e^{-x} \mathrm{~d} x, s>0$ is the Gamma function, we have

$$
\begin{aligned}
\frac{J_{n}(0)}{J_{0}(0)} & =\frac{(n+1)(n+2)}{2} \frac{B\left(\frac{3}{2}, \frac{n+2}{n}\right)}{B\left(\frac{3}{2}, \frac{2}{n}\right)}=\frac{(n+1)(n+2)}{2} \frac{\frac{\Gamma\left(\frac{3}{2}\right) \Gamma\left(\frac{n+2}{n}\right)}{\Gamma\left(\frac{3}{2}+\frac{n+2}{n}\right)}}{\frac{\Gamma\left(\frac{3}{2}\right) \Gamma\left(\frac{2}{n}\right)}{\Gamma\left(\frac{3}{2}+\frac{2}{n}\right)}} \\
& =\frac{(n+1)(n+2)}{2} \frac{\frac{2}{n}}{\frac{3}{2}+\frac{2}{n}}=\frac{2(n+1)(n+2)}{3 n+4} .
\end{aligned}
$$

The proof is completed.

Lemma 3. $\lim _{k \rightarrow k_{n}-0} \frac{J_{n}(k)}{J_{0}(k)}=n+1$.

Proof.

$$
\lim _{k \rightarrow k_{n}-0} \frac{J_{n}(k)}{J_{0}(k)}=\lim _{k \rightarrow k_{n}-0} \frac{\int_{\alpha}^{\beta} \psi^{n} E(\psi) \mathrm{d} \psi}{\int_{\alpha}^{\beta} E(\psi) \mathrm{d} \psi}=\lim _{\psi \rightarrow \pm \sqrt[n]{n+1}} \psi^{n}=n+1 .
$$

We will represent $Q$ by $J_{m}, m=0,1, \ldots, n$ in Lemma $4-9$. At first, consider the case of $n$ is even and $n$ is odd respectively.

\subsection{The case of $n$ is even}

Let us study the basic properties of $J_{2 l}, l=0,1, \ldots, n / 2$ by the following two lemmas.

Lemma 4. Let

$$
\begin{aligned}
& J=\left(J_{0}, J_{2}, \ldots, J_{n}\right)^{\mathbb{T}}, \quad J^{\prime}=\left(J_{0}^{\prime}, J_{2}^{\prime}, \ldots, J_{n}^{\prime}\right)^{\mathbb{T}}, \\
& F=\frac{2 n(n+1)(n+2) k}{(n+4)(3 n+4)}, \quad G=-\frac{4 n(n+1)(n+2)}{(n+4)(3 n+4)}, \\
& M=2(n+2) k, \quad N=-2 n .
\end{aligned}
$$


Then we have $J=\Lambda(k) J^{\prime}$, where

$$
\Lambda(k)=\left(\begin{array}{cccccc}
\frac{M}{n+4} & \frac{N}{n+4} & & & & \\
\vdots & \frac{M}{n+8} & \frac{N}{n+8} & & & \\
\vdots & \vdots & \frac{M}{n+12} & \frac{N}{n+12} & & \\
\vdots & \vdots & & \ddots & \ddots & \\
\vdots & \vdots & & & \frac{M}{3 n} & \frac{N}{3 n} \\
F & G & \cdots & \cdots & \cdots & \frac{M}{3 n+4}
\end{array}\right)_{\frac{n+2}{2} \times \frac{n+2}{2}}
$$

Proof. From the relation

$$
E^{2}=\psi^{2}-\frac{2}{(n+1)(n+2)} \psi^{n+2}-k,
$$

we get

$$
2 E \frac{\mathrm{d} E}{\mathrm{~d} \psi}=2 \psi-\frac{2}{n+1} \psi^{n+1} .
$$

$J_{0}$ can be calculated as follows.

$$
\begin{aligned}
J_{0} & =\int E \mathrm{~d} \psi=\int E^{2} \frac{\mathrm{d} \psi}{E}=\int\left(\psi^{2}-\frac{2}{(n+1)(n+2)} \psi^{n+2}-k\right) \frac{\mathrm{d} \psi}{E} \\
& =\int\left(\psi\left(\psi-\frac{2}{n+2}\left(\psi-E \frac{\mathrm{d} E}{\mathrm{~d} \psi}\right)\right)-k\right) \frac{\mathrm{d} \psi}{E} \\
& =\frac{n}{n+2} \int \frac{\psi^{2}}{E} \mathrm{~d} \psi+\frac{2}{n+2} \int \psi \mathrm{d} E-k \int \frac{\mathrm{d} \psi}{E} \\
& =2 k J_{0}^{\prime}-\frac{2 n}{n+2} J_{2}^{\prime}-\frac{2}{n+2} J_{0},
\end{aligned}
$$

i.e.,

$$
J_{0}=\frac{M}{n+4} J_{0}^{\prime}+\frac{N}{n+4} J_{2}^{\prime} .
$$

Similarly,

$$
\begin{aligned}
J_{2} & =\int \psi^{2} E \mathrm{~d} \psi=\int \psi^{2}\left(\psi\left(\frac{n}{n+2} \psi+\frac{2}{n+2} E \frac{\mathrm{d} E}{\mathrm{~d} \psi}\right)-k\right) \frac{\mathrm{d} \psi}{E} \\
& =2 k J_{2}^{\prime}-\frac{2 n}{n+2} J_{4}^{\prime}-\frac{6}{n+2} J_{2},
\end{aligned}
$$

i.e.,

$$
J_{2}=\frac{M}{n+8} J_{2}^{\prime}+\frac{N}{n+8} J_{4}^{\prime} .
$$

In the same manner, $J_{4}, J_{6}, \ldots, J_{n-2}$ can be calculated. And we get

$$
J_{4}=\frac{M}{n+12} J_{4}^{\prime}+\frac{N}{n+12} J_{6}^{\prime}, \ldots, \quad J_{n-2}=\frac{M}{3 n} J_{n-2}^{\prime}+\frac{N}{3 n} J_{n}^{\prime} .
$$


Now let us calculate $J_{n}$.

$$
\begin{aligned}
J_{n} & =\int \psi^{n} E \mathrm{~d} \psi=\int \psi^{n}\left(\psi\left(\frac{n}{n+2} \psi+\frac{2}{n+2} E \frac{\mathrm{d} E}{\mathrm{~d} \psi}\right)-k\right) \frac{\mathrm{d} \psi}{E} \\
& =2 k J_{n}^{\prime}-\frac{2(n+1)}{n+2} \int \psi^{n} E \mathrm{~d} \psi+\frac{n}{n+2} \int(n+1) \psi\left(\psi-E \frac{\mathrm{d} E}{\mathrm{~d} \psi}\right) \frac{\mathrm{d} \psi}{E} \\
& =2 k J_{n}^{\prime}-\frac{2(n+1)}{n+2} J_{n}-\frac{2 n(n+1)}{n+2} J_{2}^{\prime}+\frac{n(n+1)}{n+2} J_{0} .
\end{aligned}
$$

This together with (4.2) implies

$$
J_{n}=F J_{0}^{\prime}+G J_{2}^{\prime}+\frac{M}{3 n+4} J_{n}^{\prime} .
$$

The proof is completed.

Lemma 5. For simplicity, we write $\Lambda(k)$ as $\Lambda$. Let

$$
V=M^{\frac{n+2}{2}}+(-1)^{\frac{n+2}{2}}(n+1) M N^{\frac{n}{2}}, \quad W=\prod_{j=1}^{\frac{n+2}{2}}(n+4 j) .
$$

Then we have

$$
|\Lambda|=\frac{V}{W}, \quad \Lambda^{-1}=\frac{\Omega}{V}, \quad \Omega=\left(\omega_{i j}\right)_{\frac{n+2}{2} \times \frac{n+2}{2}} .
$$

In $(4.4)$, the $\left(\omega_{i j}\right)$ are given as follows:

$$
\begin{aligned}
& \omega_{11}=(n+4) M^{\frac{n}{2}}+(-1)^{\frac{n+2}{2}} 2(n+1)(n+2) N^{\frac{n}{2}}, \\
& \omega_{1 j}=(-1)^{1+j}(n+4 j) M^{\frac{n+2(1-j)}{2}} N^{j-1}, \quad 2 \leq j \leq(n+2) / 2, \\
& \omega_{i j}=(-1)^{i+j}(n+4 j) M^{\frac{n+2(i-j)}{2}} N^{j-i}, \quad 2 \leq i \leq j \leq(n+2) / 2, \\
& \omega_{i 1}=(-1)^{\frac{n+2 i}{2}} n(n+1) M^{i-1} N^{\frac{n+2(1-i)}{2}}, \quad 2 \leq i \leq(n+2) / 2, \\
& \omega_{i j}=(-1)^{\frac{n+2 i+2 j}{2}}(n+4 j)(n+1) M^{i-j} N^{\frac{n+2(j-i)}{2}}, \quad 2 \leq j<i \leq(n+2) / 2 .
\end{aligned}
$$

Proof. The proof can be completed by direct calculations.

\subsection{The case of $n$ is odd}

Let us study the basic properties of $J_{m}, m=0,1, \ldots, n$ by the following two lemmas.

Lemma 6. Let

$$
\tilde{J}=\left(J_{0}, J_{1}, \ldots, J_{n}\right)^{\mathbb{T}}, \quad \tilde{J}^{\prime}=\left(J_{0}^{\prime}, J_{1}^{\prime}, \ldots, J_{n}^{\prime}\right)^{\mathbb{T}} .
$$


Then we have $\tilde{J}=\tilde{\Lambda}(k) \tilde{J}^{\prime}$, where

$$
\tilde{\Lambda}(k)=\left(\begin{array}{ccccccc}
\frac{M}{n+4} & 0 & \frac{N}{n+4} & & & & \\
\vdots & \frac{M}{n+6} & 0 & \frac{N}{n+6} & & & \\
\vdots & \vdots & \frac{M}{n+8} & 0 & \frac{N}{n+8} & & \\
\vdots & \vdots & & \ddots & \ddots & \ddots & \\
\vdots & \vdots & & & \frac{M}{3 n} & 0 & \frac{N}{3 n} \\
\vdots & \frac{(n+1) N}{3 n+2} & \cdots & \cdots & \cdots & \frac{M}{3 n+2} & 0 \\
F & 0 & G & \cdots & \cdots & \cdots & \frac{M}{3 n+4}
\end{array}\right)_{(n+1) \times(n+1)}
$$

Proof. Similar to the proof of Lemma 4, we have

$$
\begin{aligned}
& J_{1}=\frac{M}{n+6} J_{1}^{\prime}+\frac{N}{n+6} J_{3}^{\prime}, \quad J_{3}= \frac{M}{n+10} J_{3}^{\prime}+\frac{N}{n+10} J_{5}^{\prime}, \\
& \ldots, \quad J_{n-3}=\frac{M}{3 n-2} J_{n-3}^{\prime}+\frac{N}{3 n-2} J_{n-1}^{\prime} .
\end{aligned}
$$

Therefore, we only need to prove

$$
J_{n-1}=\frac{M}{3 n+2} J_{n-1}^{\prime}+\frac{(n+1) N}{3 n+2} J_{1}^{\prime} .
$$

Now let us calculate $J_{n-1}$.

$$
\begin{aligned}
J_{n-1} & =\int \psi^{n-1} E \mathrm{~d} \psi=\int \psi^{n-1}\left(\psi\left(\frac{n}{n+2} \psi+\frac{2}{n+2} E \frac{\mathrm{d} E}{\mathrm{~d} \psi}\right)-k\right) \frac{\mathrm{d} \psi}{E} \\
& =2 k J_{n-1}^{\prime}-\frac{2 n}{n+2} \int \psi^{n-1} E \mathrm{~d} \psi+\frac{n}{n+2} \int(n+1)\left(\psi-E \frac{\mathrm{d} E}{\mathrm{~d} \psi}\right) \frac{\mathrm{d} \psi}{E} \\
& =2 k J_{n-1}^{\prime}-\frac{2 n}{n+2} J_{n-1}-\frac{2 n(n+1)}{n+2} J_{1}^{\prime}
\end{aligned}
$$

The proof is completed.

Lemma 7. Let

$$
\tilde{V}=M^{n+1}+(n+1)^{2} M N^{n}, \quad \tilde{W}=\prod_{j=2}^{n+2}(n+2 j) .
$$

Then we have

$$
|\tilde{\Lambda}|=\frac{\tilde{V}}{\tilde{W}}, \quad \tilde{\Lambda}^{-1}=\frac{\tilde{\Omega}}{\tilde{V}}, \quad \tilde{\Omega}=\left(\tilde{\omega}_{i j}\right)_{(n+1) \times(n+1)} .
$$


In $(4.5)$, the $\left(\tilde{\omega}_{i j}\right)$ are given as follows:

$$
\begin{aligned}
& \tilde{\omega}_{11}=(n+4) M^{n}+2(n+1)^{2}(n+2) N^{n}, \\
& \tilde{\omega}_{2 l, 1}=(-1)^{\frac{n-1}{2}-l} n(n+1) M^{\frac{n-1}{2}+l} N^{\frac{n+1}{2}-l}, \quad 1 \leq l \leq(n+1) / 2, \\
& \tilde{\omega}_{2 l+1,1}=(-1)^{l} n(n+1)^{2} M^{l} N^{n-l}, \quad 1 \leq l \leq(n-1) / 2, \\
& \tilde{\omega}_{1,2 m+1}=(-1)^{m}(n+4 m+4) M^{n-m} N^{m}, \quad 1 \leq m \leq(n-1) / 2, \\
& \tilde{\omega}_{2 l+1,2 m+1}=(-1)^{m+l}(n+4 m+4) M^{n+l-m} N^{m-l}, \quad 1 \leq l \leq m \leq(n-1) / 2, \\
& \tilde{\omega}_{2 l, 2 m}=(-1)^{m+l}(n+4 m+2) M^{n+l-m} N^{m-l}, \quad 1 \leq l \leq m \leq(n+1) / 2, \\
& \tilde{\omega}_{2 l+1,2 m+1}=(-1)^{m+l-1}(n+4 m+4)(n+1)^{2} M^{l-m} N^{n+m-l}, \\
& \quad 2 \leq l \leq(n-1) / 2,1 \leq m \leq l-1, \\
& \tilde{\omega}_{2 l, 2 m}=(-1)^{m+l-1}(n+4 m+2)(n+1)^{2} M^{l-m} N^{n+m-l}, \\
& \quad 2 \leq l \leq(n+1) 2,1 \leq m \leq l-1, \\
& \tilde{\omega}_{2 l, 2 m+1}=(-1)^{\frac{n+1}{2}-m-l}(n+4 m+4)(n+1) M^{\frac{n-1}{2}-m+l} N^{\frac{n+1}{2}+m-l}, \\
& \quad 1 \leq l \leq(n+1) / 2,1 \leq m \leq(n-1) / 2, \\
& \quad 0 \leq l \leq(n-1) / 2,1 \leq m \leq(n+1) / 2 .
\end{aligned}
$$

Proof. The proof can be completed by direct calculations.

\subsection{The representation of $Q$}

Noting the expression of $Q$ in (4.1), so at first, we have to represent $J_{n+2}$ and $J_{2 n+2}$ by $J_{m}, m=0,1, \ldots, n$. In fact, it is not difficult to obtain the following result.

Lemma 8. Let

$$
\begin{aligned}
& O=-\frac{(n+1)(n+2) k}{3 n+8}, \quad P=\frac{4(n+1)(n+2)}{3 n+8}, \quad U=-\frac{(n+1)^{2}(n+2) k}{5 n+8} . \\
& S=-\frac{(n+1)^{2}(n+2)^{2}(n+4) k}{(3 n+8)(5 n+8)}, \quad T=\frac{4(n+1)^{2}(n+2)^{2}(n+4)}{(3 n+8)(5 n+8)},
\end{aligned}
$$

Then we have

$$
J_{n+2}=O J_{0}+P J_{2} \quad \text { and } \quad J_{2 n+2}=S J_{0}+T J_{2}+U J_{n}
$$

Proof. $\quad J_{n+2}$ and $J_{2 n+2}$ can be calculated similarly to the proof of Lemma 4. Note that

$$
J_{n+2}=\int \psi^{n+2} E \mathrm{~d} \psi=\int(n+1) \psi\left(\psi-E \frac{\mathrm{d} E}{\mathrm{~d} \psi}\right) E \mathrm{~d} \psi
$$




$$
\begin{aligned}
& =(n+1) J_{2}-(n+1) \int \psi\left(\psi^{2}-\frac{2}{(n+1)(n+2)} \psi^{n+2}-k\right) \mathrm{d} E \\
& =(n+1) J_{2}-(n+1) k J_{0}+(n+1) \int\left(3 \psi^{2}-\frac{2(n+3)}{(n+1)(n+2)} \psi^{n+2}\right) E \mathrm{~d} \psi \\
& =-(n+1) k J_{0}+4(n+1) J_{2}-\frac{2(n+3)}{(n+2)} J_{n+2} .
\end{aligned}
$$

This implies that the first identity of (4.6) is true. On the other hand, we have

$$
\begin{aligned}
J_{2 n+2}= & \int \psi^{2 n+2} E \mathrm{~d} \psi=\int(n+1) \psi^{n+1}\left(\psi-E \frac{\mathrm{d} E}{\mathrm{~d} \psi}\right) E \mathrm{~d} \psi \\
= & (n+1) J_{n+2}-(n+1) \int \psi^{n+1}\left(\psi^{2}-\frac{2}{(n+1)(n+2)} \psi^{n+2}-k\right) \mathrm{d} E \\
= & (n+1) J_{n+2}+(n+1) \\
& \times \int\left((n+3) \psi^{n+2}-\frac{2(2 n+3)}{(n+1)(n+2)} \psi^{2 n+2}-(n+1) k \psi^{n}\right) E \mathrm{~d} \psi \\
= & -(n+1)^{2} k J_{n}+(n+1)(n+4) J_{n+2}-\frac{2(2 n+3)}{(n+2)} J_{2 n+2},
\end{aligned}
$$

which implies that the second identity of(4.6) is true. The proof is completed.

Lemma 9. Let us represent $Q$ by $J_{m}, m=0,1, \ldots, n$. Then we have

$$
Q=J_{n}-J_{0} .
$$

Proof. Applying (4.1) and Lemma 8, we obtain

$$
\begin{aligned}
Q= & -2 J_{2}^{\prime}+\frac{4}{n+1} J_{n+2}^{\prime}-\frac{2}{(n+1)^{2}} J_{2 n+2}^{\prime} \\
= & -2 J_{2}^{\prime}+\frac{4}{n+1}\left(\frac{O}{k} J_{0}+O J_{0}^{\prime}+P J_{2}^{\prime}\right) \\
& -\frac{2}{(n+1)^{2}}\left(\frac{S}{k} J_{0}+\frac{U}{k} J_{n}+S J_{0}^{\prime}+T J_{2}^{\prime}+U J_{n}^{\prime}\right) \\
= & \left(\frac{4}{(n+1) k} O-\frac{2}{(n+1)^{2} k} S\right) J_{0}-\frac{2}{(n+1)^{2} k} U J_{n}-\frac{2}{(n+1)^{2}} U J_{n}^{\prime} \\
& +\left(\frac{4}{n+1} O-\frac{2}{(n+1)^{2}} S\right) J_{0}^{\prime}+\left(-2+\frac{4}{n+1} P-\frac{2}{(n+1)^{2}} T\right) J_{2}^{\prime} .
\end{aligned}
$$

(1) If $n$ is even, by Lemma 5, we have

$$
\begin{aligned}
& J_{0}^{\prime}=\frac{1}{V}\left(\omega_{11} J_{0}+\omega_{12} J_{2}+\cdots+\omega_{1, \frac{n+2}{2}} J_{n}\right), \\
& J_{2}^{\prime}=\frac{1}{V}\left(\omega_{21} J_{0}+\omega_{22} J_{2}+\cdots+\omega_{2, \frac{n+2}{2}} J_{n}\right), \\
& J_{n}^{\prime}=\frac{1}{V}\left(\omega_{\frac{n+2}{2}, 1} J_{0}+\omega_{\frac{n+2}{2}, 2} J_{2}+\cdots+\omega_{\frac{n+2}{2}, \frac{n+2}{2}} J_{n}\right) .
\end{aligned}
$$


Substituting these equations into (4.7), we get $Q=C_{0} J_{0}+C_{2} J_{2}+\cdots+C_{n} J_{n}$. Now, let us calculate the coefficients $C_{0}, C_{2}, \ldots, C_{n}$. By substituting $\omega_{i j}$ into $C_{0}, C_{2}, \ldots, C_{n}$, we obtain

$$
\begin{aligned}
C_{0}= & \frac{4}{(n+1) k} O-\frac{2}{(n+1)^{2} k} S+\left(\frac{4}{n+1} O-\frac{2}{(n+1)^{2}} S\right) \frac{\omega_{11}}{V} \\
& +\left(-2+\frac{4}{n+1} P-\frac{2}{(n+1)^{2}} T\right) \frac{\omega_{21}}{V}-\frac{2}{(n+1)^{2}} U \frac{\omega_{\frac{n+2}{2}, 1}}{V}=-1, \\
C_{2 l}= & \left(\frac{4}{n+1} O-\frac{2}{(n+1)^{2}} S\right) \frac{\omega_{1, l+1}}{V} \\
& +\left(-2+\frac{4}{n+1} P-\frac{2}{(n+1)^{2}} T\right) \frac{\omega_{2, l+1}}{V}-\frac{2}{(n+1)^{2}} U \frac{\omega_{\frac{n+2}{2}, l+1}}{V} \\
= & 0, \quad \text { for } l=1,2, \ldots \frac{n-2}{2}, \\
C_{n}= & -\frac{2}{(n+1)^{2} k} U+\left(\frac{4}{n+1} O-\frac{2}{(n+1)^{2}} S\right) \frac{\omega_{1, \frac{n+2}{2}}}{V} \\
& +\left(-2+\frac{4}{n+1} P-\frac{2}{(n+1)^{2}} T\right) \frac{\omega_{2, \frac{n+2}{2}}}{V}-\frac{2}{(n+1)^{2}} U \frac{\omega_{\frac{n+2}{2}, \frac{n+2}{2}}}{V}=1 .
\end{aligned}
$$

(2) If $n$ is odd, by Lemma 7 , we have

$$
\begin{aligned}
J_{0}^{\prime} & =\frac{1}{\tilde{V}}\left(\tilde{\omega}_{11} J_{0}+\tilde{\omega}_{12} J_{1}+\cdots+\tilde{\omega}_{1, n+1} J_{n}\right), \\
J_{2}^{\prime} & =\frac{1}{\tilde{V}}\left(\tilde{\omega}_{21} J_{0}+\tilde{\omega}_{22} J_{1}+\cdots+\tilde{\omega}_{2, n+1} J_{n}\right), \\
J_{n}^{\prime} & =\frac{1}{\tilde{V}}\left(\tilde{\omega}_{n+1,1} J_{0}+\tilde{\omega}_{n+1,2} J_{1}+\cdots+\tilde{\omega}_{n+1, n+1} J_{n}\right) .
\end{aligned}
$$

Substituting these equations into (4.7), we get $Q=\tilde{C}_{0} J_{0}+\tilde{C}_{1} J_{1}+\cdots+\tilde{C}_{n} J_{n}$. Now, let us calculate the coefficients $\tilde{C}_{0}, \tilde{C}_{1}, \ldots, \tilde{C}_{n}$. By substituting $\tilde{\omega}_{i j}$ into $\tilde{C}_{0}, \tilde{C}_{1}, \ldots, \tilde{C}_{n}$, we obtain

$$
\begin{aligned}
\tilde{C}_{0}= & \frac{4}{(n+1) k} O-\frac{2}{(n+1)^{2} k} S+\left(\frac{4}{n+1} O-\frac{2}{(n+1)^{2}} S\right) \frac{\tilde{\omega}_{11}}{\tilde{V}} \\
& +\left(-2+\frac{4}{n+1} P-\frac{2}{(n+1)^{2}} T\right) \frac{\tilde{\omega}_{21}}{\tilde{V}}-\frac{2}{(n+1)^{2}} U \frac{\tilde{\omega}_{n+1,1}}{\tilde{V}}=-1, \\
\tilde{C}_{m}= & \left(\frac{4}{n+1} O-\frac{2}{(n+1)^{2}} S\right) \frac{\tilde{\omega}_{1, m+1}}{\tilde{V}} \\
& +\left(-2+\frac{4}{n+1} P-\frac{2}{(n+1)^{2}} T\right) \frac{\tilde{\omega}_{2, m+1}}{\tilde{V}}-\frac{2}{(n+1)^{2}} U \frac{\tilde{\omega}_{n+1, m+1}}{\tilde{V}} \\
= & 0, \quad \text { for } m=1,2, \ldots, n-1, \\
\tilde{C}_{n}= & \frac{2}{(n+1)^{2} k} U+\left(\frac{4}{n+1} O-\frac{2}{(n+1)^{2}} S\right) \frac{\tilde{\omega}_{1, n+1}}{\tilde{V}} \\
+ & \left(-2+\frac{4}{n+1} P-\frac{2}{(n+1)^{2}} T\right) \frac{\tilde{\omega}_{2, n+1}}{\tilde{V}}-\frac{2}{(n+1)^{2}} U \frac{\tilde{\omega}_{n+1, n+1}}{\tilde{V}}=1 .
\end{aligned}
$$

The proof is completed. 


\subsection{The proof of Proposition 1 and Theorem 1}

Now, for arbitrary integer $n>0$, we investigate the property of

$$
X=\frac{Q}{R}=\frac{J_{n}}{J_{0}}-1
$$

Let $\tilde{X}=\frac{J_{n}}{J_{0}}$ and $Z=\frac{J_{n}^{\prime}}{J_{0}^{\prime}}$. Then we have the following three lemmas.

Lemma 10. If $\tilde{X}^{\prime}\left(k^{*}\right)=0$ for some $0<k^{*}<k_{n}$, then

$$
\frac{2(n+1)(n+2)}{3 n+4}<\tilde{X}\left(k^{*}\right)<n+1 .
$$

Proof. By Lemma 4 or 6 , we have

$$
\begin{aligned}
& J_{0}=\frac{2(n+2) k}{n+4} J_{0}^{\prime}-\frac{2 n}{n+4} J_{2}^{\prime}, \\
& J_{n}=\frac{2 n(n+1)(n+2) k}{(n+4)(3 n+4)} J_{0}^{\prime}-\frac{4 n(n+1)(n+2)}{(n+4)(3 n+4)} J_{2}^{\prime}+\frac{2(n+2) k}{3 n+4} J_{n}^{\prime} .
\end{aligned}
$$

Eliminating $J_{2}^{\prime}$ yields

$$
\frac{2(n+1)(n+2)}{3 n+4} J_{0}-J_{n}=\frac{2(n+1)(n+2) k}{3 n+4} J_{0}^{\prime}-\frac{2(n+2) k}{3 n+4} J_{n}^{\prime},
$$

i.e.,

$$
\frac{J_{n}}{J_{0}}-\frac{2(n+1)(n+2)}{3 n+4}=\frac{2(n+2) k}{(3 n+4) J_{0}} J_{0}^{\prime}\left(\frac{J_{n}^{\prime}}{J_{0}^{\prime}}-(n+1)\right) .
$$

If $\tilde{X}^{\prime}\left(k^{*}\right)=0$, then

$$
\tilde{X}\left(k^{*}\right)=Z\left(k^{*}\right)=\frac{J_{n}^{\prime}\left(k^{*}\right)}{J_{0}^{\prime}\left(k^{*}\right)},
$$

and

$$
\tilde{X}\left(k^{*}\right)-\frac{2(n+1)(n+2)}{3 n+4}=\frac{2(n+2) k^{*}}{3 n+4} \frac{J_{0}^{\prime}\left(k^{*}\right)}{J_{0}\left(k^{*}\right)}\left(\tilde{X}\left(k^{*}\right)-(n+1)\right) .
$$

Since $J_{0}^{\prime}\left(k^{*}\right) / J_{0}\left(k^{*}\right)<0$, we have

$$
\left(\tilde{X}\left(k^{*}\right)-(n+1)\right)\left(\tilde{X}\left(k^{*}\right)-\frac{2(n+1)(n+2)}{3 n+4}\right)<0,
$$

and the proof is completed.

Combining Lemma 2, 3 and 10, we get the following lemma.

Lemma 11. For $0 \leq k<k_{n}$, we have

$$
\frac{2(n+1)(n+2)}{3 n+4} \leq \tilde{X}(k) \leq n+1 .
$$


By noting the relation (4.8), this proves Proposition 1.

Let $c_{0}$ be $c_{0}=X(k)^{-1}$. Then from Proposition 1, we have

Lemma 12. For $0 \leq k<k_{n}$, the pairs $\left(\psi_{0}, c_{0}\right)$ satisfies the limit speed condition (3.8) for periodic wave and solitary wave solutions with $\alpha=\alpha(k)$. Moreover,

$$
\frac{1}{n} \leq c_{0}(k) \leq \frac{3 n+4}{2 n^{2}+3 n}, \quad c_{0}(0)=\frac{3 n+4}{2 n^{2}+3 n}, \quad \lim _{k \rightarrow k_{n}-0} c_{0}(k)=\frac{1}{n} .
$$

Since

$$
\frac{\partial \tilde{\Phi}}{\partial c}\left(\alpha(k), c_{0}, 0\right)=\frac{1}{2 \sqrt{c_{0}}} \int \psi_{0}^{\prime \prime 2} \mathrm{~d} \tau+\frac{1}{2 c_{0} \sqrt{c_{0}}} \int \psi_{0}^{\prime 2} \mathrm{~d} \tau>0
$$

and similarly $\partial \tilde{\Psi} / \partial c>0$, we can solve the equation $\tilde{\Phi}=0$ and $\tilde{\Psi}=0$ by the implicit function theorem. More precisely, there exists a unique smooth function $c_{k}(\varepsilon)=c(\varepsilon, k)$ for each $k \in\left[0, k_{n}\right)$ and $\varepsilon \in\left(0, \varepsilon_{n}^{*}\right)$ such that

$$
\begin{gathered}
\tilde{\Phi}(\alpha(k), c(\varepsilon, k), \varepsilon)=0 \text { for } 0<k<k_{n}, 0<\varepsilon<\varepsilon_{n}^{*}, \\
\tilde{\Psi}(c(\varepsilon, k), \varepsilon)=0 \text { for } 0<\varepsilon<\varepsilon_{n}^{*} .
\end{gathered}
$$

Note that $k=2 h$ and $k_{n}=2 h_{n}$. Therefore, we get Theorem 1 .

\section{Conclusions}

In this work we have considered a perturbed generalized KdV equation. Using the geometric singular perturbation theory and the regular perturbation analysis for a Hamitonian system, we have proved that solitary wave solutions and periodic wave solutions persist for sufficiently small perturbation parameter. Moreover, the upper and lower bounds of the limit wave speed are obtained.

For $n=1$, Ogawa [17] proved that $c_{0}(k)$ is a smooth decreasing function, but for arbitrary integer $n>0$, this problem is still under consideration.

\section{References}

[1] M.J. Ablowitz and P.A. Clarkson. Solitons, Nonlinear Evolution Equations and Inverse Scattering. 2nd edition, Cambridge University Press, 1992.

[2] M.J. Ablowitz and H. Segur. Solitons and the Inverse Scattering Transform. Society for Industrial and Applied Mathematics, 1981.

[3] J.L. Bona, S. Vento and F.B. Weissler. Singularity formation and blowup of complex-valued solutions of the modified KdV equation. Discrete Contin. Dyn. Syst., 33:4811-4840, 2013. http://dx.doi.org/10.3934/dcds.2013.33.4811.

[4] L. Escauriaza, C.E. Kenig, G. Ponce and L. Vega. On uniqueness properties of solutions of the $k$-generalized KdV equations. J. Funct. Anal., 244(2):504-535, 2007. http://dx.doi.org/10.1016/j.jfa.2006.11.004.

[5] X. Fan and L. Tian. The existence of solitary waves of singularly perturbed mKdV-KS equation. Chaos Soliton Fractals, 26(4):1111-1118, 2005.

http://dx.doi.org/10.1016/j.chaos.2005.02.014. 
[6] N. Fenichel. Geometric singular perturbation theory for ordinary differential equations. J. Differ. Equations, 31(1):53-98, 1979. http://dx.doi.org/10.1016/0022-0396(79)90152-9.

[7] Y. Fu and Z. Liu. Existence of travelling wavefronts of KdV-Burgers equation. Appl. Math. Lett., 24:897-900, 2011.

http://dx.doi.org/10.1016/j.aml.2010.12.047.

[8] C.S. Gardner, J.M. Greene, M.D. Kruskal and R.M. Miura. Method for solving the Korteweg-de Vries equation. Phys. Rev. Lett., 19(1):1095-1097, 1967. http://dx.doi.org/10.1103/PhysRevLett.19.1095.

[9] C. Gu, H. Hu and Z. Zhou. Darboux Transformations in Integrable Systems: Theory and Their Applications to Geometry. Springer-Verlag, New York, 2005.

[10] M.A. Helal and M.S. Mehanna. A comparative study between two different methods for solving the general Korteweg-de Vries equation (GKdV). Chaos Soliton Fractals, 33(3):725-739, 2007. http://dx.doi.org/10.1016/j.chaos.2006.11.011.

[11] E. Infeld and G. Rowlands. Nonlinear Waves, Solitons and Chaos. Cambridge University Press, 2000.

[12] C.K.R.T. Jones. Geometric singular perturbation theory. Dyn. Syst., pp. 44-118, 1995.

[13] E.L. Korotyaev. Estimates for solutions of KDV on the phase space of periodic distributions in terms of action variables. Discrete Contin. Dyn. Syst., 30(7):219225, 2011. http://dx.doi.org/10.3934/dcds.2011.30.219.

[14] S. Kutluaya and Y. Ucara. A quadratic B-spline Galerkin approach for solving a coupled KdV equation. Math. Model. Anal., 18(1):103-121, 2013. http://dx.doi.org/10.3846/13926292.2013.760011.

[15] Z. Liu and C. Yang. The application of bifurcation method to a higher-order kdv equation. J. Math. Anal. Appl., 275(1):1-12, 2002. http://dx.doi.org/10.1016/S0022-247X(02)00210-X.

[16] Y. Martel and F. Merle. Refined asymptotics around solitons for gKdV equations. Discrete Contin. Dyn. Syst., 20(2):177-218, 2008. http://dx.doi.org/10.3934/dcds.2008.20.177.

[17] T. Ogawa. Travelling wave solutions to a perturbed Korteweg-de Vries equation. Hiroshima Math. J., 24(2):401-422, 1994.

[18] T. Ogawa. Periodic travelling waves and their modulation. Jpn. J. Ind. Appl. Math., 18(2):521-542, 2001. http://dx.doi.org/10.1007/BF03168589.

[19] Y. Tang, W. Xu, J. Shen and L. Gao. Persistence of solitary wave solutions of singularly perturbed Gardner equation. Chaos Soliton Fractals, 37(2):532-538, 2008. http://dx.doi.org/10.1016/j.chaos.2006.09.044. 